Crumpton, M. J. \& Wilkinson, J. M. (1963). Biochem. J.88, 228.

Fraenkel-Conrat, H. \& Porter, R. R. (1952). Biochim. biophys. Acta, $\mathbf{9}, \mathbf{5 5 7}$.

Fraenkel-Conrat, H., Snell, N. S. \& Ducay, E. D. (1952a). Arch. Biochem. Biophys. 39, 80.

Fraenkel-Conrat, H., Snell, N. S. \& Ducay, E. D. (1952b). Arch. Biochem. Biophys. 39, 97.

François, C., Marshall, R. D. \& Neuberger, A. (1962). Biochem. J. 83, 335.

Green, N. M. (1963a). Biochem. J. 89, 585.

Green, N. M. (1963b). Biochem. J. 89, 599.

Green, N. M. (1963c). Biochem. J. 89, 609.

Hirs, C. W. H., Moore, S. \& Stein, W. H. (1953). J. biol. Chem. 200, 493.

Jacobs, S. (1960). Analyst, 85, 257.

Jacobs, S. (1962). Analyst, 87, 53.

Johansen, P. G., Marshall, R. D. \& Neuberger, A. (1960). Biochem. J. 77, 239.
Kraan, J. G. \& Muir, H. (1957). Biochem. J. 66, 55 P.

Marshall, R. D. \& Neuberger, A. (1960). Nature, Lond., 186, 311.

Moore, S. (1963). J. biol. Chem. 238, 235.

Moore, S. \& Stein, W. H. (1954). J. biol. Chem. 211, 907.

Moore, S. \& Stein, W. H. (1956). Advanc. Protein Chem. 11, 191.

Rhodes, M. B., Azari, P. R. \& Feeney, R. E. (1958). J. biol. Chem. 230, 399.

Rondle, C. J. M. \& Morgan, W. T. J. (1955). Biochem. J. 61, 586.

Sober, H. A., Gutter, F. J., Wyckoff, M. M. \& Peterson, E. A. (1956). J. Amer. chem. Soc. 78, 756.

Tallan, H. M. \& Stein, W. H. (1953). J. biol. Chem. 200, 507.

Tracey, M. V. (1952). Biochem. J. 52, 265.

Warren, L. (1959). J. biol. Chem. 234, 1971.

Winzler, R. J. (1955). Meth. biochem. Anal. 2, 279.

Biochem. J. (1963) 89. 599

\title{
Avidin
}

\section{THE NATURE OF THE BIOTIN-BINDING SITE}

\author{
By N. M. GREEN* \\ Department of Chemical Pathology, St Mary's Hospital Medical School, London, W. 2
}

(Received 19 March 1963)

Green (1963 $a$ ) and Melamed \& Green (1963) have shown that each molecule of avidin binds three molecules of biotin and that the complex has a low dissociation constant of about $10^{-15} \mathrm{M}$. The present paper is concerned with the nature of the binding site.

Previous work on this subject has been confined to (1) microbiological studies by Wright and his coworkers on the binding of biotin analogues (for a summary see György, 1954), and (2) studies of the effect of chemical modification of avidin on its activity (Fraenkel-Conrat, Snell \& Ducay, $1952 b$ ). The first group of workers showed that the ureido ring of biotin is of great importance for firm binding, but that substitution of the carboxyl group or modification of the thiophan ring has a relatively small effect. They also showed that of the available stereoisomers of D-biotin only DL-epiallobiotin, epimeric at $\mathrm{C}_{(4)}$, is bound by avidin. Some of these findings have now been confirmed and extended by more exact measurements of dissociation constants, particularly of weakly bound analogues such as urea and ethyleneurea (imidazolid-2-one). Previous evidence for binding of the latter compounds had

* Present address: Department of Chemistry, National Institute for Arthritis and Metabolic Diseases, Bethesda 14, Md., U.S.A. been provided by experiments of Fraenkel-Conrat et al. (1952b) which showed that they stabilized avidin against acid or alkaline denaturation. The experiments with chemical modifications were relatively inconclusive in that, though many of the reagents produced slight inactivation, only prolonged esterification, oxidation with hydrogen peroxide $-\mathrm{Fe}^{2+}$ ions and treatment with formaldehyde in the presence of an amide led to more than $60 \%$ inactivation. The sensitivity to hydrogen peroxide is probably due to the same groups that are responsible for sensitivity to photo-oxidation (György, Rose \& Tomarelli, 1942), and both this and the inactivation by formaldehyde could have been due to modification of tryptophan residues. However, this point only became clear from spectroscopic and chemical evidence presented in a preliminary note (Green, 1962). In this it was shown that binding of biotin produces a red shift of the tryptophan absorption bands of avidin which can readily be detected by the resultant difference spectrum. The large difference extinction coefficient at $233 \mathrm{~m} \mu(25000 /$ mole of biotin bound) provides a useful method for the determination of avidin or biotin by spectrophotometric titration. Biotin analogues, even distantly related ones, gave similar but smaller spectral shifts that have now 
been used to make a comparative study of analoguebinding.

The importance of tryptophan in the biotinbinding reaction was confirmed by oxidizing avidin with $N$-bromosuccinimide (Green, 1962), which showed both that biotin protected the tryptophan from oxidation and that destruction of the tryptophan in avidin led to its inactivation. These results are now presented in more detail.

\section{MATERIALS}

Avidin was prepared by the method of Melamed \& Green (1963)

D-Biotin (Roche Products Ltd.) was recrystallized from hot water. Biotin sulphone and the diamino carboxylic acid [8-(3,4-diaminothiophan-2-yl)pentanoic acid] resulting from alkaline hydrolysis of biotin were prepared by the methods of Hofmann, Melville \& Du Vigneaud (1941). Biotin anilide was prepared by the method of Wolf, Valiant \& Folkers (1951). $N^{\alpha}$-Acetyl-L-tryptophan was prepared by the method of Greenstein \& Winitz (1961).

Ethyleneurea was treated with charcoal and recrystallized from methanol.

Succinimide was recrystallized from water. $N$-Bromosuccinimide was recrystallized from acetic acid and contained $97 \%$ of the theoretical amount of active bromine.

Ethylene glycol (glycol) was redistilled.

The methyl esters of $N^{1}$ - and $N^{3}$-methoxycarbonylbiotin were kindly provided by Dr J. Knappe.

Other materials were commercial products, used without further purification.

Biotinyl-e-aminohexanoic acid. This was prepared from the acid chloride of biotin and methyl $\epsilon$-aminohexanoate by using the method of Wolf et al. (1951) for biotinyl- $\beta$-alanine. Biotin acid chloride (from $\mathbf{0} \cdot \mathbf{2}$ g. of biotin) was shaken with methyl $\epsilon$-aminohexanoate (2 g.) and allowed to stand overnight. The reaction mixture was taken up in $20 \mathrm{ml}$. of chloroform and washed twice with $0.5 \mathrm{~N}-\mathrm{HCl}$, then with $0 \cdot 5 \mathrm{M}-\mathrm{NaHCO}_{3}$ and finally with water. The chloroform layer was dried over anhydrous $\mathrm{MgSO}_{4}$ and evaporated almost to dryness. The amorphous residue was extracted with $3 \times 9 \mathrm{ml}$. of boiling ethyl acetate. The amorphous precipitates, which came down on cooling, were filtered. The yield was $68 \mathrm{mg}$., m.p. 132-136 ${ }^{\circ}$. The ester was hydrolysed by treatment with $1 \mathrm{ml}$. of $0.3 \mathrm{~N}-\mathrm{NaOH}$ in $50 \%(\mathrm{v} / \mathrm{v})$ methanol for $20 \mathrm{~min}$. at $60^{\circ}$. The methanol was removed in vacuo and the residual solution acidified with $3 \mathrm{~N}-\mathrm{HCl}$. The white precipitate was filtered and washed with water. The yield was $50 \mathrm{mg}$., m.p. 212-216 ${ }^{\circ}$. Spectrophotometric titration showed that it neutralized $95 \%$ of the theoretical amount of avidin (Found: $\mathrm{C}, 54 \cdot 1 ; \mathrm{H}, 7 \cdot 8 ; \mathrm{N}, 11 \cdot 1$. Calc. for $\mathrm{C}_{16} \mathrm{H}_{27} \mathrm{~N}_{3} \mathrm{O}_{4} \mathrm{~S}: \mathrm{C}, 53 \cdot 8 ; \mathrm{H}, 7 \cdot 6 ; \mathrm{N}, 11 \cdot 8 \%$ ).

Dinitrophenyl derivative of biotin hydrazide. A portion (60 mg.) of biotin hydrazide (Hofmann, Melville \& Du Vigneaud, 1942) was dissolved in $10 \mathrm{ml}$. of $50 \%(\mathrm{v} / \mathrm{v})$ ethanol plus $1 \mathrm{ml}$. of $1 \mathrm{~m}-\mathrm{NaHCO}_{3} ; 1 \mathrm{ml}$. of a $10 \%(\mathrm{v} / \mathrm{v})$ solution of 1-fluoro-2,4-dinitrobenzene in ethanol was added slowly with shaking. After standing for $2 \mathrm{hr}$. at room temperature the deep-red solution was diluted with $20 \mathrm{ml}$. of water and extracted with $3 \times 40 \mathrm{ml}$. of ether. Acidification of the aqueous layer with $2 \mathrm{~N}$-acetic acid gave yelloworange microcrystals, which were centrifuged and then filtered with suction. The material was recrystallized from $50 \%(\mathrm{v} / \mathrm{v})$ ethanol and then from $90 \%(\mathrm{v} / \mathrm{v})$ methanol. The yield was $20 \mathrm{mg}$., m.p. $224^{\circ}$. The substance was slow to dissolve and slow to crystallize. The $\lambda_{\max }$. was $341 \mathrm{~m} \mu(\epsilon=$ $12500)$. In alkaline solution the colour changed to red and the absorption band shifted to $404 \mathrm{~m} \mu(\epsilon=18000)$. It neutralized $97 \%$ of the theoretical amount of avidin as measured by spectrophotometric titration (Found: C, 45.0; $\mathrm{H}, 5 \cdot 0 ; \mathrm{N}, 19 \cdot 2$. Calc. for $\mathrm{C}_{16} \mathrm{H}_{20} \mathrm{~N}_{6} \mathrm{O}_{6} \mathrm{~S}: \mathrm{C}, 45 \cdot 3 ; \mathrm{H}, 4 \cdot 8 ; \mathrm{N}$, $19 \cdot 8 \%$ ).

\section{METHODS}

Difference spectra and spectrophotometric titrations. Measurements were made on a Unicam SP. 700 double-beam recording spectrophotometer. Solutions were prepared directly in cuvettes by using Carlsberg constriction pipettes (H. E. Pedersen, 37 Peter Bangsvej, Copenhagen F, Denmark) for small volumes and grade A graduated pipettes for volumes greater than $0.5 \mathrm{ml}$. Two cuvettes were inserted in each spectrophotometer compartment, one of each pair containing avidin and the other containing solvent. In most experiments the base-line (avidin minus avidin) was checked at this stage. The perturbing solute was then added in equal small volumes to the avidin in the sample beam and to the solvent cuvette in the reference beam. The same volume of solvent was added to the other two cuvettes. These additions were made with Carlsberg pipettes or with Agla micrometer syringes. By repeating this process a spectrophotometric titration could be carried out at any desired wavelength and the complete difference spectrum could be determined at any stage during the titration. The contents of the cuvettes were mixed, either by gentle agitation or by stirring with small glass-enclosed magnets. The observed extinction differences were corrected for dilution during the titration. In experiments on the difference spectrum induced by biotin it was usually unnecessary to employ tandem cells since the extinction due to biotin was negligible above $210 \mathrm{~m} \mu$. Care was taken to avoid stray-light errors by keeping the total extinction in any one beam below 2 , or, where the highest accuracy was required, below 1-5. A check of Beer's law, by using lysozyme at $233 \mathrm{~m} \mu$, where many of the measurements were performed, showed about $1 \%$ departure at $E=1 \cdot 5,3 \%$ at $E=2$ and $10 \%$ at $E=3$. Such errors will be magnified in measurements of difference spectra, where the reference sample is usually of high extinction.

Titrations were carried out between 229 and $233 \mathrm{~m} \mu$ depending on the wavelength of maximum difference, which varied slightly from one analogue to another. The results are expressed as $\Delta E_{\max .} / E_{282}$, where $\Delta E_{\max }$ is the extinction change at the maximum of the difference spectrum. The value of this ratio at the equivalence point can be converted into a difference extinction coefficient/mole of analogue bound by multiplying by $\epsilon_{282} / 3$ for avidin $\left(2 \cdot 8 \times 10^{4}\right)$. The value of $E_{333} / \mathrm{mg}$. of avidin/ml. was $5 \cdot 3$. The spectral shift was sometimes calculated from $\Delta E$ by using the relation

$$
\Delta E=\frac{\mathrm{d} E !}{\mathrm{d} \lambda} \cdot \Delta \lambda
$$

The value of $\mathrm{d} E / \mathrm{d} \lambda$ at the appropriate wavelength was calculated directly from recorded spectra, by using a high chart speed to decrease the slope and increase the accuracy of the measurement. 
Direct measurement of spectral shift. These were kindly performed by Dr G. H. Beaven using the logarithmic cam method of Holiday (1950) to determine the position of the tryptophan fine-structure band at about $290 \mathrm{~m} \mu$.

Oxidation with $\mathrm{N}$-bromosuccinimide. Avidin was titrated with freshly prepared $2 \mathrm{~mm}-N$-bromosuccinimide in $50 \mathrm{~mm}$ sodium acetate buffer, pH $4 \cdot 6$ (Patchornik, Lawson, Gross \& Witkop, 1960). The changes in extinction at the maximum $(282 \mathrm{~m} \mu)$ and at the fine-structure peak $(291 \mathrm{~m} \mu)$ were followed, and the amount of avidin tryptophan oxidized was calculated by using the values of $\Delta \epsilon$ found with $N$-acetyltryptophan. The maximum and fine-structure peak of the latter occur at slightly shorter wavelengths and the values of $\Delta \epsilon$ at the end point were $4.0 \times 10^{3}(279 \mathrm{~m} \mu)$ and $3.3 \times 10^{3}$ $(288 \mathrm{~m} \mu)$.

\section{RESULTS}

Difference spectrum of the avidin-biotin complex. When three molar equivalents of biotin were added to avidin the spectral bands were shifted to the red. The difference spectrum between the complex and avidin at the same concentration is shown in Fig. 1. The general appearance of the spectrum is very similar to that observed when tryptophan or $\mathrm{N}$-acetyltryptophan is dissolved in ethylene glycol, guanidine hydrochloride, or other solvent of high refractive index (Fig. 1; and Bigelow \& Geschwind, 1960). The only marked difference was the shoulder at about $300 \mathrm{~m} \mu$ which is absent from tryptophan difference spectra. The relative magnitudes of $\Delta \epsilon_{\max }$. in the $230 \mathrm{~m} \mu$ and $290 \mathrm{~m} \mu$ regions were approximately the same for both spectra. This is consistent with the hypothesis that tryptophan is responsible for the $233 \mathrm{~m} \mu$ difference peak of the avidin difference spectrum. The calculated values of $\Delta \lambda$ are compared in Table 1 with an experimental value obtained by direct measurement of the position of the fine-structure peak at about $290 \mathrm{~m} \mu$. Although there is a considerable difference between the results obtained by the two methods it is probably not much greater than the combined experimental errors.

The appearance of the difference spectrum hardly changed with $\mathrm{pH}$ between 2 and 13, and the titration of avidin with biotin, by using the $233 \mathrm{~m} \mu$ peak, could be carried out anywhere in this region (Fig. 2 and Table 2). The slightly larger value of $\Delta E_{233} / E_{282}$ in alkali was due to concomitant changes in tyrosine ionization which is discussed by Green $(1963 b)$. The linear relation between spectral shift and added biotin persisted right up to the end point. This indicates negligible dissociation of the complex at a concentration of $10 \mu \mathrm{M}$, as would be expected from the kinetic data given by Green $(1963 a)$. The linear relationship also confirms the validity of Beer's law and the absence of stray-light errors. The end point was reached after the addition of 1 mole of biotin/17 700 g. of avidin, showing that there are probably three binding sites/molecule (mol.wt. approx. 60 000; Fraenkel-Conrat, Snell \& Ducay, 1952a). Since the equivalent weight could be determined with considerable accuracy, a molecular weight of $53000(3 \times 17700)$ has been used throughout the present series of papers.

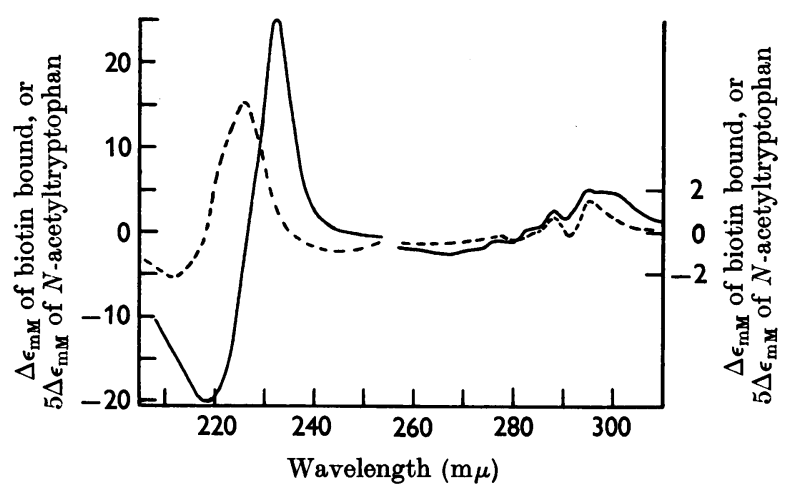

Fig. 1. Comparison of difference spectra: —-, avidinbiotin complex minus avidin, in $50 \mathrm{~mm}$-sodium phosphate, pH 6.8 $\left(\Delta \epsilon_{\mathrm{mx}}\right.$ of biotin bound $) ;-\cdots . \cdot, N$-acetyltryptophan in $5 \mathrm{M}$-glycol made $0 \cdot 1 \mathrm{~N}$ with respect to $0 \cdot 1 \mathrm{~N}-\mathrm{HCl}$ minus $N$-acetyltryptophan in $0 \cdot 1 \mathrm{~N}-\mathrm{HCl}\left(\Delta \epsilon_{\mathrm{mm}}\right.$ has been multiplied by 5 for comparison with the biotin-binding unit, which contains $4 \cdot 7$ tryptophan residues). The ordinate scale above $255 \mathrm{~m} \mu$ has been expanded by a factor of 2.5 . The glycolinduced difference spectrum in this region has been replotted with a $3 \mathrm{~m} \mu$ red shift to facilitate comparison with the avidin-biotin spectrum. This shift is due to the different reference cells used for the two spectra. That for the glycol spectrum contained $N$-acetyltryptophan, whereas that for the biotin spectrum contained avidin, whose tryptophan fine-structure peak is shifted $3 \mathrm{~m} \mu$ to the red relative to $N$-acetyltryptophan (Green, $1963 b$ ).

Table 1. Spectral shifts induced by biotin and by glycol

Details are given in the text.

$\begin{array}{lc}\text { Absorbing species } & \text { Agent inducing } \\ \text { Avidin } & \text { the shift } \\ & \text { Biotin }\end{array}$

$N$-Acetyltryptophan Glycol (6 M)
Method
Difference spectrum Difference spectrum Logarithmic cam Difference spectrum Difference spectrum

$\begin{array}{cc}\lambda & \Delta \lambda \\ (\mathrm{m} \mu) & (\AA) \\ 233 & 15 \\ 295 & 8 \\ 291 & 5 \\ 226 & 9 \\ 292 & 5\end{array}$


Attempts were made to follow the reaction spectrophotometrically by using a primitive stopped-flow apparatus (Green, 1963 $a$ ), discharging into a $10 \mathrm{~cm}$. cuvette. With this long light-path it

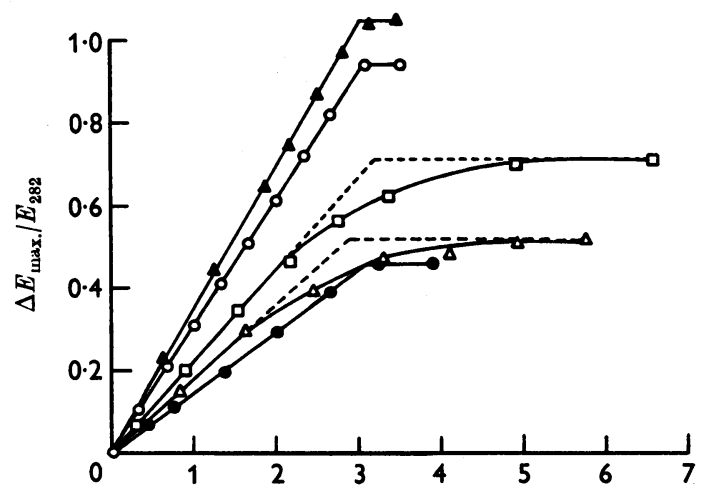

Biotin analogue added (moles/mole of avidin)

Fig. 2. Spectrophotometric titration of avidin $(4 \mu \mathrm{M})$ with biotin and closely related analogues. $O$, Biotin (in $50 \mathrm{~mm}$ sodium phosphate, $\mathrm{pH} \mathrm{6.8);} \Delta$, biotin (in $0.5 \mathrm{~N}-\mathrm{KOH}$ ); $\square$, diamine from biotin (in 50 mM-sodium phosphate, $\mathrm{pH} \mathrm{6.8)}$; $\triangle, N^{1 '}$-methoxycarbonylbiotin methyl ester (in $50 \mathrm{mN}-$ ammonia-acetic acid buffer, $\mathrm{pH} 4.6)$; 9 , biotin DNPhydrazide (in $50 \mathrm{mN}$-ammonia-acetic acid buffer, pH 4.6). was possible to work at a concentration of $0.3 \mu \mathrm{M}$, but even at this concentration the reaction was at least $90 \%$ complete between 5 and 10 sec. after mixing. This would mean that the second-order rate constant must be greater than $10^{6}-10^{7} \mathrm{M}^{-1} \mathrm{sec}^{-1}$, in agreement with results obtained with $\left[{ }^{14} \mathrm{C}\right]$ biotin under the same conditions of $\mathrm{pH}$ and ionic strength (Green, 1963a).

Titration of avidin with biotin analogues. Avidin was titrated spectrophotometrically with biotin and a number of analogues by using the difference maximum in the $233 \mathrm{~m} \mu$ region as a measure of complex formation. The results for firmly bound compounds are shown in Fig. 2 and Table 2 (compounds 1-9). Those analogues (compounds 2-5) in which either the terminal carboxyl group of biotin had been substituted or the thiophan ring modified gave no evidence of dissociation. The magnitude of the spectral shift was less than that found with biotin and this enables one to follow the displacement of the analogue by biotin. When biotin was added to these avidin-biotin analogue complexes at the end of a titration no further spectral shift was observed during $1 \mathrm{hr}$. Thus less than $5-10 \%$ of the analogue was released in this time, in contrast with the release of the more weakly bound compounds discussed below. It seems therefore that the close analogues must be bound almost as firmly as biotin.

Table 2. Binding of biotin analogues by avidin

Details are given in the text. The values of low dissociation constants $\left(<10^{-6} \mathbf{M}\right) \cdot$ were calculated from the amount of free avidin present at the equivalence point, which was determined from the titration curves. The smallest measurable value of $K$ was about $10^{-8} \mathrm{M}$. Larger values of $K$ were obtained (1) approximately, from the analogue concentration required for half the maximum effect, or (2) accurately, by fitting the results to a curve calculated from the law of mass action.

1. Biotin

Biotin

Biotin

2. Biotin sulphone

3. Biotinyl- $\epsilon$-aminohexanoate

4. Biotin anilide

5. Biotin DNP-hydrazide

6. $N^{1^{\prime}}$-Methoxycarbonylbiotin methyl ester

7. $N^{3}$-Methoxycarbonylbiotin methyl ester

8. Diamine from biotin [ $\delta$-(3,4-diaminothiophan-2. yl)pentanoic acid]

9. DL-Lipoate

10. Ethyleneurea

11. Urea $(1 \cdot 5 \mathrm{M})$

12. Hexanoate

13. Decanoate

14. $\gamma$-Cyclohexylbutyrate

15. DL- $\beta$-Phenylalanine

16. Succinimide

17. Ethylene glycol $(5 \mathrm{M})$

18. Guanidine hydrochloride (3M)

\begin{tabular}{ll}
$\lambda_{\max }$ & $\Delta E_{\max }$ \\
\cline { 2 - 2 }$(\mathrm{m} \mu)$ & $E_{282}$ \\
233 & $0 \cdot 94$ \\
233 & $1 \cdot 05$ \\
232 & $0 \cdot 9$ \\
233 & $0 \cdot 84$ \\
233 & $0 \cdot 76$ \\
$232 \cdot 5$ & $0 \cdot 68$ \\
$232 \cdot 5$ & $0 \cdot 46$ \\
- & $0 \cdot 43$ \\
$232 \cdot 5$ & $0 \cdot 52$ \\
- & $0 \cdot 34$ \\
$232 \cdot 5$ & $0 \cdot 45$ \\
- & - \\
233 & $0 \cdot 73$ \\
$233 \cdot 5$ & $0 \cdot 39$ \\
233 & $0 \cdot 26$ \\
233 & $0 \cdot 33$ \\
229 & $0 \cdot 16$ \\
229 & $0 \cdot 11$ \\
$232 \cdot 5$ & $0 \cdot 20$ \\
- & 0 \\
$\overline{229}$ & 0 \\
230 & $0 \cdot 44$ \\
& $0 \cdot 17$
\end{tabular}

$10^{-15 *}$

$<10^{-8}$

$<10^{-8}$

$<10^{-8}$

$<10^{-8}$

$<10^{-8}$

$<10^{-8}$

$7 \times 10^{-6}$

$4 \times 10^{-7}$

$1.5 \times 10^{-8}$

$<10^{-8}$

$10^{-2}$

$3 \times 10^{-7}$

$7 \times 10^{-7}$

$5 \times 10^{-4}$

$3.6 \times 10^{-2}$

$3 \times 10^{-4}$

$1 \times 10^{-5}$

$1 \times 10^{-4}$

$>0 \cdot 1$

$>0 \cdot 1$

$0 \cdot \overline{1}$ 
Analogues (compounds 6-9) in which the ureido ring is modified or absent were bound much less firmly. The diamine (compound 8) showed a dissociation constant of $3 \times 10^{-7} \mathrm{M}$, which, although low, was greater than that for biotin by a factor of at least $10^{7}$. The addition of biotin to this complex immediately displaced the analogue with a rise of $\Delta E_{233} / E_{232}$ to $0 \cdot 94$. The isomeric $N$-methoxycarbonylbiotin methyl esters (compounds 6 and 7), in which the ureido ring was again modified, were somewhat similar. The $N^{1^{\prime}}$-methoxycarbonyl isomer, possibly related to the $N$-carboxybiotinyl-enzymes (Lynen et al. 1961), was the less firmly bound, though the dissociation constant $\left(4 \times 10^{-i} \mathrm{M}\right)$ was still so low that inhibition of the transformations of carboxylated biotinyl-enzymes by avidin should be observable. The $N^{3 \prime}$-methoxycarbonyl isomer, whose dissociation was only just detectable by this method, differed from the other weakly bound analogues in that its displacement from avidin by biotin was slow enough to be followed spectrophotometrically. The rate-limiting step was presumably the dissociation of the complex and the first-order rate constant at $\mathrm{pH} 4 \cdot 6$ was 0.03 sec. $^{-1}$. Lipoic acid is another analogue of some biochemical interest, which resembles biotin without the ureido ring. It was bound by avidin with a dissociation constant of less than $10^{-6} \mathrm{M}$ and it should therefore be possible to inhibit lipovl-onzymes reversibly with avidin provided that the lipoyl residue is sufficiently exposed. Although no effect of $\mathrm{pH}$ (over the range 3-13) on the titration of avidin with biotin could be observed, this was probably due to the impossibility of detecting changes in the low dissociation constant by this method. When analogues were studied it was observed that a lowering of the $\mathrm{pH}$ to 4.6 often increased the dissociation constant (compounds 6-8).

The results of titrating avidin with a number of weakly bound analogues are shown in Fig. 3. and Table 2 (compounds 10-18). These are of two main types: urea and ethyleneurea, resembling the ureido ring of biotin, and a number of mediumchain-length fatty acids which bear some resemblance to the remainder of the biotin molecule. The titration curves for urea and ethyleneurea closely followed those predicted from the law of mass action for binding at a single type of site. Although the number of these cannot be determined from the present data it seems likely that three/molecule are involved in view of the similarity of the difference spectrum to that induced by biotin. This relationship between the binding sites was further confirmed by the.effect of biotin added at the end of the titration. The final $\Delta E_{\max .} / E_{282}$ observed in such experiments was 0.94 for imidazolid-2-one and 1.08 for urea $(2 \cdot 8 \mathrm{M})$, showing that the effect of biotin is competitive and not additive. The slight additional red shift $(0 \cdot 14)$ in urea was due to the increased refractive index of the medium (Bigelow \& Geschwind, 1960). This interpretation is confirmed by the approximately linear increase with urea concentration long after the specific binding sites have been saturated (Fig. 3). This continued up to urea concentrations of $8 \mathrm{M}$. Most proteins would be denatured in this solvent and would show a denaturation blue shift (Bigelow \& Geschwind, 1960), but avidin appears to be stable, and its behaviour in this respect is discussed in more detail by Green $(1963 b)$. In contrast with ethyleneurea, succinimide did not produce a spectral shift even at a concentration of $30 \mathrm{~mm}$. This provides further evidence for specific interaction of the ureido group with avidin.

The results with carboxylic acids followed approximately the law of mass action at low concentrations, but at higher concentrations (greater than $2 \mathrm{mM}$ ) there was an apparent drop in the base-line of the difference spectrum which made interpretation difficult. The results for these compounds (Table 1) are therefore only approximate. The difference peak was at a shorter wavelength than usual $(229 \mathrm{~m} \mu)$ which may indicate that the binding was not strictly analogous to that of biotin and the more closely related analogues.

A number of other organic molecules (e.g. ethanol and pyridine) unrelated in structure to biotin also gave small red shifts when added in relatively high concentrations $(0 \cdot 1-1 \mathrm{M})$. This is probably a con-

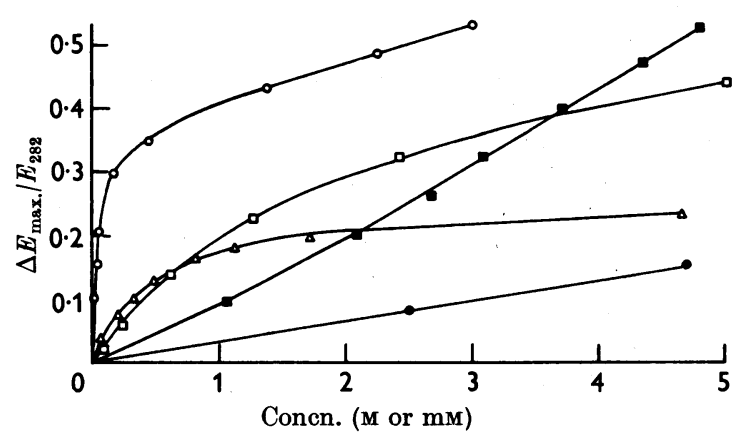

Fig. 3. Spectral shifts of avidin $(4 \mu \mathrm{M})$ induced by ethyleneurea $(\triangle$; abscissa units, $\mathrm{mm})$ and urea $(O$; abscissa units, M); and spectral shifts of avidin ( $\square$ ), avidin-biotin complex (O) and $N$-acetyltryptophan ( $\square$ ) induced by ethylene glycol (abscissa units, $\mathrm{M}$ ). All experiments were performed in 50 mm-sodium phosphate buffer, $\mathrm{pH} 6 \cdot 8$, except for $N$-acetyltryptophan which was in $0 \cdot 1 \mathrm{~N}-\mathrm{HCl}$. The continuous curves shown for urea and ethyleneurea were calculated from the law of mass action, by using the dissociation constants shown in Table 2. The curve for urea also contains a term linear in urea concentration to allow for the non-specific red shift (see the text). This term was obtained by a linear extrapolation from the points at high concentrations of urea. 
sequence of the non-polar nature of the binding site but insufficient systematic work has been done to allow any firm conclusion at present.

Spectral shifts induced by glycol. The effect of ethylene glycol on the spectrum was studied in some detail because it has been suggested that the magnitude of the induced spectral shift is a function of the extent of exposure of the aromatic amino acid side chains to the aqueous environment (Herskovits \& Laskowski, 1962). These authors have suggested that only those residues that are on the surface of the protein, and so exposed to the solvent, will have their absorption spectra changed as the concentration of glycol is increased. They have attempted to calculate the proportion of buried and exposed groups by comparing the observed red shift with that found when the chromophore is completely exposed to solvent (e.g. in $N$-acetyltryptophan). It was therefore decided to compare avidin and the avidin-biotin complex from this point of view in order to confirm the hypothesis that combination with biotin leads to burial of the tryptophan residues and possibly to determine the number of these involved. The method is only valid if the glycol itself does not affect the protein conformation and hence possibly the extent of exposure of the tryptophan residues. This assumption appears to hold for many proteins (Simpson \& Kauzmann, 1953), but it was necessary to check the method for avidin in view of the variety of different substances with which it could interact. This was done by following the concentration-dependence of the shift in the usual way. If the extent of exposure remained unchanged by the glycol, an approximately linear relation between spectral shift and glycol concentration should result. Further, the maximum slope should be less than that found with $N$-acetyltryptophan in glycol. Fig. 3 shows that the relationship was not linear and that the initial slope was greater than that observed with $N$-acetyltryptophan. The high slope indicates some positive interaction between the avidin and the glycol resulting in partial burial of the tryptophan residues. The general effect was rather similar to that produced by increasing concentrations of urea, although the difference maximum occurred at $229 \mathrm{~m} \mu$; in addition about 20 times the concentration of glycol was required to give half the maximum effect. The curve did not follow that calculated from the law of mass action for association at a single type of binding site and no quantitative interpretation has been attempted. The effect of glycol on the avidin-biotin complex was relatively small (Fig. 3) and linear up to at least $9 \mathrm{M}$-glycol. It does not appear that an interpretation in terms of number of tryptophan residues exposed in the complex can be made since the difference spectrum was not typical. The sharp peak at $229 \mathrm{~m} \mu$ was replaced by a broad hump extending below $220 \mathrm{~m} \mu$, and the peaks and troughs in the $280 \mathrm{~m} \mu$ region were hardly detectable. However, this small effect is consistent with the tryptophan-burial hypothesis.

Oxidation of tryptophan residues in avidin. In most proteins that have been studied the tryptophan residues were completely oxidized by titration with $N$-bromosuccinimide (Witkop, 1961) and the $280 \mathrm{~m} \mu$ peak was replaced by a shoulder at $250 \mathrm{~m} \mu$. By analogy with the products given by simple indole derivatives it was concluded that this shoulder is due to the formation of a substituted oxindole. Spectrophotometric titration of avidin with $N$-bromosuccinimide followed a similar course to that observed with many other proteins. The addition of each portion of $N$-bromosuccinimide was accompanied by an immediate decrease in $E_{232}$ until an end point was reached (Green, 1962). However, the oxidation was not complete since the spectrum of the product showed inflexions at $282 \mathrm{~m} \mu$ and at $291 \mathrm{~m} \mu$, which indicates that some of the tryptophan was still intact. The extent of oxidation was calculated from the change in extinction at both 282 and $291 \mathrm{~m} \mu$. Four different avidin preparations gave similar results. Out of 14 tryptophan residues $12 \cdot 4 \pm 0.2(282 \mathrm{~m} \mu)$ or $12 \cdot 0 \pm 0 \cdot 2$ $(291 \mathrm{~m} \mu)$ were destroyed; thus two were relatively resistant to the action of $N$-bromosuccinimide. Altogether 2.5 moles of $N$-bromosuccinimide were consumed/tryptophan residue oxidized, rather more than was required by $N$-acetyltryptophan, but of the same order as that required by a number of other proteins (Witkop, 1961).

Titration of the avidin-biotin complex gave different results (Green, 1962; and Fig. 4). There was no immediate decrease in $E_{282}$ after the addition of $N$-bromosuccinimide, though long standing did lead to some destruction of tryptophan. For example, 5 moles of $N$-bromosuccinimide $/ \mathrm{mole}$ of tryptophan brought about a $30 \%$ decrease of $E_{282}$ during $30 \mathrm{~min}$. Combination with biotin did not therefore provide complete protection of the tryptophan though it diminished the rate of oxidation by several orders of magnitude. The binding of each biotin protected four tryptophan residues/ molecule from the action of $N$-bromosuccinimide. To correlate the oxidation with the biotin-binding activity a series of avidin samples were treated with increasing amounts of $N$-bromosuccinimide. The extent of oxidation was determined spectrophotometrically, the binding of $\left[{ }^{14} \mathrm{C}\right]$ biotin was followed by using carboxymethylcellulose (Green, 1963a) and each sample was titrated spectrophotometrically with biotin. The results (Fig. 4) showed a slight change in the relation between tryptophan oxidized and $N$-bromosuccinimide added after about $25 \%$ of the tryptophan had been oxidized, 
suggesting that the first portions of $N$-bromosuccinimide produced slightly more oxidation than the later ones. The oxidation of $25 \%$ of the tryptophan results in the loss of about $26 \%$ of the biotinbinding capacity of the avidin, but the oxidation of the next $25 \%$ (when a total of $50 \%$ of the tryptophan is oxidized) brings about a total loss of ability to combine with biotin. Spectrophotometric titration of partially oxidized avidin gave the curves

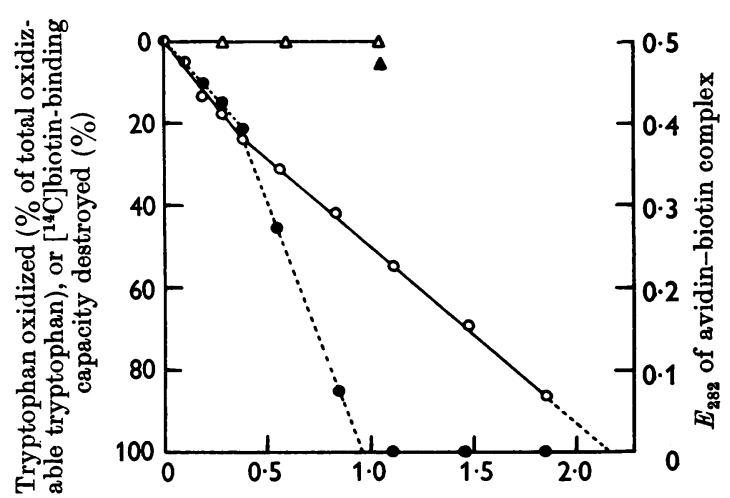

$N$-Bromosuccinimide added (moles/mole of tryptophan)

Fig. 4. Effect of $N$-bromosuccinimide at $\mathrm{pH} 4 \cdot 6$ on avidin $(O, O)$ and on the avidin-biotin complex $(\Delta, \Delta)$. The lefthand ordinates $(\%)$ refer to the avidin and the right-hand ordinates $\left(E_{282}\right)$ to the avidin-biotin complex. O, Oxidized tryptophan as a percentage of total oxidizable tryptophan $(12 \cdot 2$ out of 14 tryptophan residues/mole); 9 , percentage of $\left[{ }^{14} \mathrm{C}\right]$ biotin-binding capacity destroyed; $\triangle, E_{282}$ of avidin-biotin complex, read within $1 \mathrm{~min}$. of the addition of $N$-bromosuccinimide; $\boldsymbol{\Delta}, E_{282}$ of avidin-biotin complex, after standing for 15 min. with $N$-bromosuccinimide.

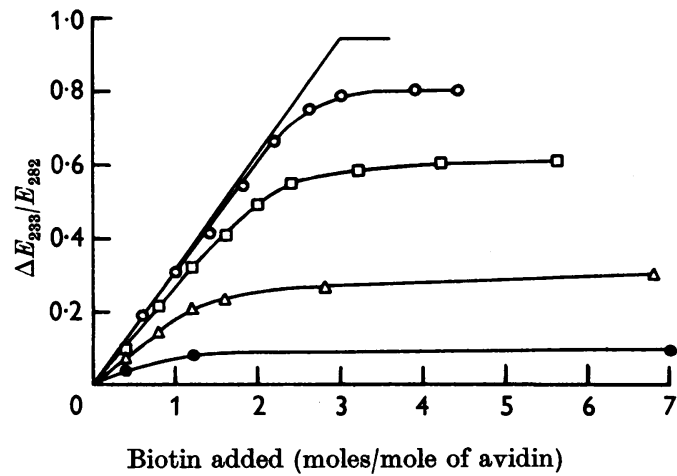

Fig. 5. Spectrophotometric titration of partially oxidized avidin with biotin, in $50 \mathrm{~mm}$-sodium acetate buffer, $\mathrm{pH} \mathrm{4 \cdot 6.}$ Each curve corresponds to one of the points of Fig. 3 and is designated by the percentage of oxidizable tryptophan which has been destroyed. $\Delta E_{233}$ was divided by $E_{282}$ of the original solution of unoxidized avidin. No points, $0 \%$ (taken from Fig. 2); $O, 13 \% ; \square, 23 \% ; \triangle, 42 \% ; 0,69 \%$. shown in Fig. 5. The general fall in activity with oxidation was confirmed. The curvature of the titration curves in the neighbourhood of the equivalence points indicates that some of the partially oxidized species were binding biotin relatively weakly and were partially dissociated at a concentration of $10 \mu \mathrm{M}$. This weak binding may account for the appreciable spectral shift that persisted at quite high degrees of oxidation where no biotinbinding was detected with $\left[{ }^{14} \mathrm{C}\right]$ biotin. The $\left[{ }^{14} \mathrm{C}\right]$ biotin assays were performed at a concentration of $0 \cdot 1 \mu \mathrm{M}$, where such partially oxidized complexes would be completely dissociated. Another form of avidin modified by partial oxidation was obtained when a solution of avidin was concentrated by pervaporation (Melamed \& Green, 1963). The absorption spectrum of this material, together with that of the unmodified avidin, is shown in Fig. 6 . Some idea of the absorption spectrum of the oxidized tryptophan was obtained by adding unmodified avidin to the reference cell until the characteristic tryptophan inflexion at $291 \mathrm{~m} \mu$ could no longer be detected. The resulting curve (Fig. 6, bottom line) showed a shoulder at $260 \mathrm{~m} \mu(\epsilon=$ 3400 ) and appreciable absorption as far as $340 \mathrm{~m} \mu$ $(\epsilon=1600)$. It was calculated from the spectra that about six of the 14 tryptophan residues/molecule had been converted into this unknown product. The pervaporated avidin retained $73 \%$ of its biotin-binding capacity, in contrast with the

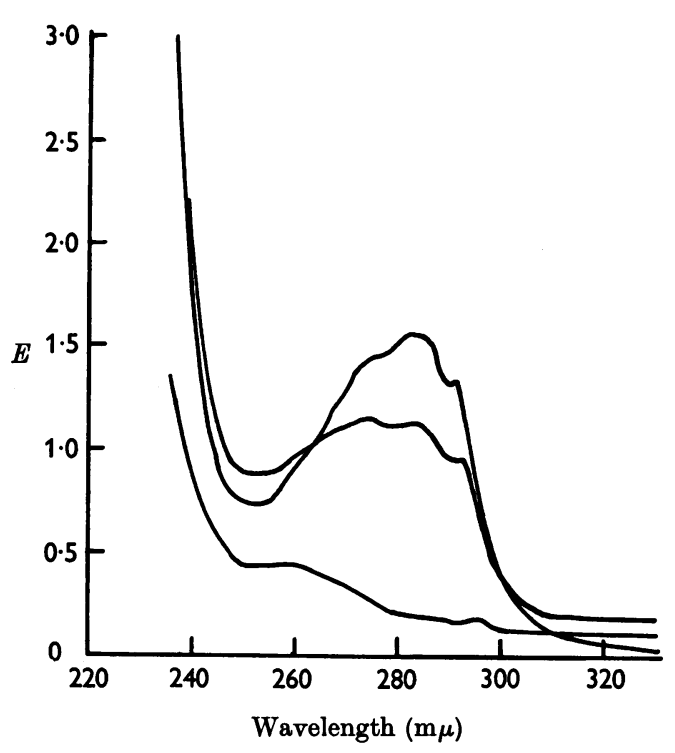

Fig. 6. Absorption spectrum of avidin (upper curve) and periodate-oxidized or pervaporated avidin (middle curve). The bottom curve was obtained by subtracting the contribution of unoxidized tryptophan from the middle curve, as described in the text. 


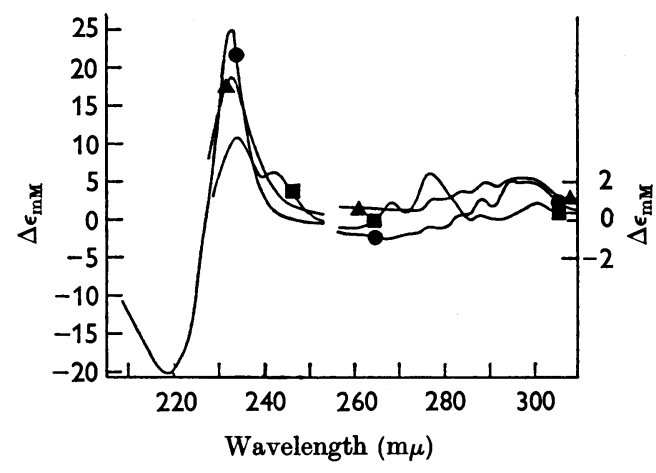

Fig. 7. Biotin-induced difference spectra of avidin and partially oxidized avidins. The ordinate scale has been expanded by a factor of 2.5 in the long-wavelength region. The values of $\Delta \epsilon$ are expressed per mole of biotin bound rather than per mole of avidin. Avidin; $\Delta, N$-bromosuccinimide-oxidized avidin $(30 \%$ of oxidizable or $26 \%$ of total tryptophan destroyed); $\square$, autoxidized or periodateoxidized avidin (40\% of total tryptophan destroyed).

$\mathrm{N}$-bromosuccinimide-oxidized material which retained only $15 \%$ of its activity when oxidized to the same extent. The difference spectra given by the two oxidized avidins were also different (Fig. 7). That given by the product of treatment with $N$. bromosuccinimide was very similar to that from unmodified avidin, apart from a raised base-line at wavelengths above $250 \mathrm{~m} \mu$. The pervaporated avidin gave three new peaks $(243,268$ and $277 \mathrm{~m} \mu)$. The first two of these corresponded to inflexion points in the spectrum of the tryptophan-oxidation product and they could have arisen from a red shift of this spectrum.

At present there is insufficient evidence to justify speculation on the nature of the oxidized tryptophan residues. However, avidin with the same absorption spectrum was also obtained by oxidation with $1 \mathrm{~mm}$-potassium periodate for $1 \mathrm{hr}$. at $\mathrm{pH} 7$. The difference spectrum produced by biotin was also the same as that given by the pervaporated product. Some of the avidin tryptophan residues must be unusually reactive since the extinctions at $280 \mathrm{~m} \mu$ of $N$-acetyltryptophan, chymotrypsinogen and lysozyme decrease by less than $5 \%$ with periodate under the same conditions. This reactivity would explain the long-known sensitivity of avidin to photo-oxidation (György et al. 1942).

\section{DISCUSSION}

Interpretation of the difference spectrum. Scheraga (1961) has discussed the various factors leading to spectral shifts in the $280 \mathrm{~m} \mu$ region of protein absorption spectra. They appear to be due either to changes in charge in the immediate neighbourhood of the chromophore, changes in hydrogen-bonding of the chromophore or changes in the polarity of the environment. There is as yet no evidence for hydrogen-bonding of the indole nitrogen in proteins and there appears to be no reason to invoke it here. The invariance of the difference spectrum with $\mathrm{pH}$ suggests that charge effects can be neglected, at least to a first approximation. The spectral shifts observed in the present paper and by Green (1963b) have therefore been explained entirely in terms of the effects of the medium, that is, in terms of stabilization of the tryptophan excited states by dipole-induced dipole interactions (Bayliss \& Macrae, 1954). In these terms the difference spectrum would be due to a biotin-induced transfer of some of the avidin tryptophan residues from a partially aqueous environment to a non-polar environment of high refractive index. This conclusion is further supported by the fact that glycol has less effect on the spectrum of the avidin-biotin complex than on the spectrum of avidin, and also by the large blue shift of the fluorescence-emission spectrum of avidin (Green, 1963c).

In the $280 \mathrm{~m} \mu$ region the only anomalous feature in the difference spectrum is the marked shoulder at $300 \mathrm{~m} \mu$, which could sometimes be seen as a separate peak when the spectrum was scanned on an expanded scale. A similar peak, observed by Bigelow \& Sonenberg (1962) when serum albumin was exposed to low concentrations of sodium dodecyl sulphate, was shown to be due to an effect on the tryptophan spectrum. The difference spectrum observed when trypsin combines with pancreatic trypsin inhibitor (N. M. Green, unpublished work; Beaven \& Holiday, 1952) is also of this type. Weber (1960) has observed anomalies in the fluorescence-polarization spectrum of proteins in this region which he has interpreted in terms of two separate electronic transitions. It is possible that these may be affected differentially by different media and so may produce variable effects in the $300 \mathrm{~m} \mu$ region.

It is not certain that the short-wavelength features of the difference spectrum can be interpreted solely in terms of a red shift of the tryptophan spectrum. For example, the results of Glazer \& Smith (1961) show that difference peaks in the $230 \mathrm{~m} \mu$ region consequent on protein denaturation are due in part to changes in peptide-bond absorption which are produced by changes in helical configuration. However, it is unlikely that such contributions are important here for the following reasons: (1) The optical rotation $\left([\alpha]_{\mathrm{D}}\right)$ is hardly changed when avidin combines with biotin (Green, $1963 b)$, suggesting that changes in helical configuration are small. (2) The peptide bonds account for only $15 \%$ of the total extinction at $233 \mathrm{~m} \mu$ 
(Green, 1962), so that small changes in their contribution can only account for a small fraction of the $25 \%$ change in extinction coefficient accompanying biotin-binding. (3) The $233 \mathrm{~m} \mu$ peak can be quantitatively accounted for by a $1.5 \mathrm{~m} \mu$ red shift of the short-wavelength tryptophan absorption band, which is the same, relative to the $290 \mathrm{~m} \mu$ shift, as that observed with $N$-acetyltryptophan in glycol (Table 1).

It is less easy to explain the trough at $212 \mathrm{~m} \mu$ in these terms, since it is much larger than the corresponding feature of the glycol-induced difference spectrum of $N$-acetyltryptophan (Fig. 1). The peptide chromophore is the major absorbing species in this region and it is possible that changes in its absorption are partly responsible for this (Rosenheck \& Doty, 1961).

Spectrophotometric titrations. Since the spectral shift induced by biotin was a linear function of the amount of biotin added, either the biotin combined more or less at random with any site, and the spectral shift was independent of the number of biotin residues bound/molecule, or there could have been a co-operative combination in which avidin molecules were saturated one by one, in which case no assumption need be made about the independence of the shift with respect to the number of biotin residues bound/molecule. Evidence in favour of the first of these alternatives is presented by Green (1963c).

The results on the affinity of avidin for various biotin analogues agree qualitatively with those previously obtained by the competition method of Wright \& Skeggs (1947). In the previous studies avidin was added to an equivalent amount of biotin in the presence of various amounts of the analogue, and the concentration of analogue required to halve the amount of biotin bound was determined. Since these experiments involve many hours of incubation during the microbiological assay, any analogue which can dissociate from the avidin during the initial stages of the incubation is liable to be almost entirely replaced by biotin, so that only those analogues that dissociate slowly will appear to compete with biotin. These included derivatives in which the carboxyl group is substituted and in which the thiophan ring is modified (Wright, Skeggs \& Cresson, 1947, 1951). However, when the ureido ring is broken no competition can be demonstrated. The present results confirm that modification or removal of the ureido ring greatly increased the dissociation constant (by a factor of $\left.10^{7}-10^{8}\right)$, but a considerable residual affinity remained. Some specific bonding of the ureido ring was also indicated by the affinity of avidin for urea and ethyleneurea. These interactions are probably mediated by hydrogen bonds. Fraenkel-Conrat et al. (1952b) suggested that a diacylimide structure might act as a complementary hydrogen-bonding site, but it is impossible to reconcile this with the known bond angles involved. The weak binding of a variety of non-polar organic molecules by avidin is possibly correlated with the red shift of the tryptophan spectrum, in that both observations suggest that part of the binding site consists of non-polar side chains.

The decreased affinity of avidin for several analogues at acid $\mathrm{pH}$ is in agreement with other evidence on its behaviour under these conditions. First, avidin undergoes reversible denaturation below pH 2 which is prevented by the prior addition of biotin (Green, 1963b). Secondly, although the avidin-biotin complex is stable at this $\mathrm{pH}$ as judged by the unchanged difference spectrum, the rate of exchange with $\left[{ }^{14} \mathrm{C}\right]$ biotin at $\mathrm{pH} 2$ (Green, $1963 a$, and unpublished work) is at least doubled. This weakening of the complex at acid $\mathrm{pH}$ is possibly due to the increased net charge on the molecule.

Oxidation of avidin. The results of the oxidation with $N$-bromosuccinimide show that four tryptophan residues are involved in each biotin-binding site, and it is likely that all of these contribute to the difference spectrum. The new biotin-induced difference peaks, which appeared when avidin was oxidized by air or periodate, suggest that the oxidation product resembles the parent tryptophan sufficiently closely for it to undergo a similar environmental change when the biotin is bound. In contrast, the oxindole type of product made no contribution to the difference spectrum (Fig. 7). [A red shift of the spectrum of free oxindole, induced by guanidine hydrochloride, produced a positive difference peak at $265 \mathrm{~m} \mu$, corresponding to the inflexion point on the long-wavelength side of the $250 \mathrm{~m} \mu$ absorption band (N. M. Green, unpublished work).] The oxindole residues, therefore, are not buried when biotin is bound by avidin that has been oxidized with $N$-bromosuccinimide. This may be due to steric differences between the oxindole and tryptophan, and such differences could have been enhanced by bromination accompanying the oxidation with $N$-bromosuccinimide (Lawson, Patchornik \& Witkop, 1960). Alternatively, cleavage of tryptophyl peptide bonds accompanying oxindole formation may have prevented participation of the oxindole in the biotin-binding process. However, in view of the generally low efficiency of the cleavage reaction (Witkop, 1961) it seems that there must be other factors involved. A more exact interpretation requires a study of the extent of cleavage accompanying oxidation and of the nature of the newly formed $N$-terminal groups. If each of the four tryptophan residues were linked by its carboxyl group to a different amino acid, it should be possible to distinguish between random and 
sequential reaction with $N$-bromosuccinimide and it might then be possible to correlate the reaction of one of the tryptophan residues with the marked loss in activity between $25 \%$ and $50 \%$ oxidation.

Nature of the binding site. An instructive analogy for tryptophan surrounded by non-polar side chains is found in myoglobin and haemoglobin. Inspection of the myoglobin model (through the courtesy of Dr H. C. Watson) showed that the tryptophan residue at position $\mathbf{A}_{15}$ was completely surrounded by non-polar side chains (five residues of valine, leucine or isoleucine). Comparison with the sequence of human haemoglobin (Watson \& Kendrew, 1961) shows a tryptophan in the same position in both $\alpha$ - and $\beta$-chains. Moreover, the homologous positions to the five surrounding nonpolar side chains of myoglobin were also occupied by valine, leucine, isoleucine or phenylalanine in both $\alpha$ - and $\beta$-chains of haemoglobin. In view of the similar three-dimensional structure of the $\alpha$ - and $\beta$-sub-units and of myoglobin (Perutz et al. 1960) it is likely that each haemoglobin molecule contains four buried tryptophan residues. This may well be correlated with the presence of four tryptophan residues/molecule in sheep and rat haemoglobins which are resistant to oxidation by $N$-bromosuccinimide at pH 4 (quoted by Witkop, 1961).

An attempt was made to estimate the contribution of such non-polar interaction to the free energy of binding. From the distribution coefficient of skatole between light petroleum and water (N. M. Green, unpublished work) a free energy of transfer of $3.2 \mathrm{kcal} . / \mathrm{mole}$ was calculated. Since the tryptophan residues in avidin are only partially exposed to the solvent, not more than about half the theoretical maximum of $13 \mathrm{kcal}$. could be obtained by transferring four of them/molecule to the non-polar interior. However, it is likely that a number of other nonpolar side chains interact with the tryptophan in the avidin-biotin complex so that a total of 10 12 kcal. could perhaps be derived from a transfer of non-polar groups from water to a non-aqueous environment. It also seems likely from much recent work, in particular that on the structure of myoglobin (Kendrew et al. 1961), that hydrophobic interactions make a large contribution to the stability of native proteins. Kauzmann (1959) has given a lucid discussion of this type of interaction where he has pointed out that the stability of a micelle-like configuration is due largely to the increased entropy of the aqueous solvent after removal of the non-polar side chains. Now, the unique configuration of a native protein, or, for example, the specificity of biotin-binding, implies a fairly sharp potential-energy minimum corresponding to a particular structure. It is difficult to account for this solely in terms of hydrophobic interactions since these are not stabilized by specific chemical bonds. However, specificity may arise where the non-polar side chains are held on a more or less rigid peptide chain or on an $\alpha$-helix. Of the restricted configurations available there may well be one, involving close packing of the side chains, which maximizes the entropy of the solvent, thus greatly increasing the stability of the system. This stability would be further enhanced by the consequent exclusion of water from access to the hydrogen bonds of an $\alpha$-helix. If one looks at the binding of biotin in these terms it may be suggested that the ureido group provides a specific but weak anchoring point and that, if the remainder of the molecule has the steric configuration of D-biotin, the side chains of the avidin can pack round it closely leading to a new conformation whose great stability is due to the increased entropy of the whole system.

\section{SUMMARY}

1. The combination of biotin with avidin produces a shift of the avidin absorption spectrum to the $\operatorname{red}(\Delta \lambda=8 \AA$ at $293 \mathrm{~m} \mu$ and $15 \AA$ at $233 \mathrm{~m} \mu)$. The resulting difference spectrum, which is characteristic of tryptophan, shows a large peak at $233 \mathrm{~m} \mu$ $\left(\Delta \epsilon_{\mathrm{mM}}\right.$ of biotin bound $\left.=25\right)$.

2. Avidin was titrated with biotin and a number of analogues by using the $233 \mathrm{~m} \mu$ peak as a measure of complex formation. Analogues in which the carboxyl group was substituted or the thiophan ring modified were firmly bound to avidin $\left(K<10^{-8} \mathrm{M}\right)$ and were not displaced by biotin. Substitution or disruption of the ureido ring greatly weakened the binding. DL-Lipoic acid. $\left(K=6 \times 10^{-7} \mathrm{M}\right)$ was an example of this type of compound. A number of simple organic substances also produced small red shifts and correspondingly weak binding $\left(K>10^{-4} \mathrm{M}\right)$.

3. The role of tryptophan in the biotin-binding mechanism was confirmed by the use of $N$ bromosuccinimide, which instantaneously oxidized 12 out of 14 tryptophan residues/molecule of avidin but did not attack any in the avidin-biotin complex unless left for long periods of time. This shows that each bound biotin protects four tryptophan residues/molecule from oxidation.

4. Oxidation of an average of one tryptophan residue out of four led to slight inactivation, but when a second tryptophan residue was destroyed the activity fell to less than $10 \%$.

5. These results have been interpreted in terms of a binding site with a specific, though weak, affinity for the ureido groups. When biotin is bound at this site it is suggested that the weak complex is stabilized by interactions between tryptophan and the non-polar side chains of the protein and of biotin. 
I thank Dr H. C. Watson for an instructive discussion on the structure of myoglobin, Dr G.H. Beaven for the measurements of the position of the tryptophan finestructure peak, and Professor A. Neuberger, F.R.S., for reading the manuscript. This work was supported by a grant from the Nuffield Foundation.

\section{REFERENCES}

Bayliss, N. S. \& Macrae, E. G. (1954). J. phys. Chem. 58, 1002.

Beaven, G. H. \& Holiday, E. R. (1952). Advanc. Protein Chem. 7, 342.

Bigelow, C. C. \& Geschwind, I. I. (1960). C.R. Lab Carlsberg, Sér. chim. 31, 283.

Bigelow, C. C. \& Sonenberg, M. (1962). Biochemistry, 1, 197.

Fraenkel-Conrat, H., Snell, N. S. \& Ducay, E. D. (1952a). Arch. Biochem. Biophys. 39, 80.

Fraenkel-Conrat, H., Snell, N. S. \& Ducay, E. D. (1952b). Arch. Biochem. Biophys. 39, 97.

Glazer, A.N. \& Smith, E. L. (1961). J. biol. Chem.236, 2942.

Green, N. M. (1962). Biochim. biophys. Acta, 59, 244.

Green, N. M. (1963a). Biochem. J. 89, 585.

Green, N. M. (1963b). Biochem. J. 89, 609.

Green, N. M. (1963c). Biochem. J. (in the Press).

Greenstein, J.P. \& Winitz, M. (1961). Chemistry of the Amino Acids, p. 2726. New York and London: John Wiley and Sons Inc.

György, P. (1954). In The Vitamins, vol. 1, p. 526. Ed. by Sebrell, W. H. \& Harris, R. S. New York: Academic Press Inc.

György, P., Rose, C. S. \& Tomarelli, R. (1942). J. biol. Chem. 144, 169.

Herskovits, T. T. \& Laskowski, M. (1962). J. biol. Chem. 237, 2481.

Hofmann, K., Melville, D. B. \& Du Vigneaud, V. (1941). J. biol. Chem. 141, 207.
Hofmann, K., Melville, D. B. \& Du Vigneaud, V. (1942). J. biol. Chem. 144, 513.

Holiday, E. R. (1950). In Analytical Absorption Spectroscopy, p. 268. Ed. by Mellon, M. G. London: Chapman and Hall Ltd.

Kauzmann, W. (1959). Advanc. Protein Chem. 14, 1.

Kendrew, J. C., Watson, H. C., Strandberg, B. E., Dickerson, R. E., Phillips, D. C. \& Shore, V. C. (1961). Nature, Lond., 190, 666.

Lawson, W. B., Patchornik, A. \& Witkop, B. (1960). J. Amer. chem. Soc. 82, 5918.

Lynen, F., Knappe, J., Lorch, E., Jutting, G., Ringelmann, E. \& Lachance, J. P. (1961). Biochem. Z. 335, 123.

Melamed, M. D. \& Green, N. M. (1963). Biochem. J. 89, 591.

Patchornik, A., Lawson, W. B., Gross, E. \& Witkop, B. (1960). J. Amer. chem. Soc. 82, 5923.

Perutz, M. F., Rossmann, M. G., Cullis, A. F., Muirhead, H., Will, G. \& North, A. C. T. (1960). Nature, Lond., 185, 416.

Rosenheck, K. \& Doty, P. (1961). Proc. nat. Acad. Sci., Wash., 47, 1775.

Scheraga, H. A. (1961). Protein Structure, p. 217. New York and London: Academic Press Inc.

Simpson, R. B. \& Kauzmann, W. (1953). J. Amer. chem. Soc. 75, 5139.

Watson, H. C. \& Kendrew, J. C. (1961). Nature, Lond., 190, 670.

Weber, G. (1960). Biochem. J. 75, 345.

Witkop, B. (1961). Advanc. Protein Chem. 16, 221.

Wolf, D. E., Valiant, J. \& Folkers, K. (1951). J. Amer. chem. Soc. 73, 4142 .

Wright, L. D. \& Skeggs, H. R. (1947). Arch. Biochem. 12 , 27.

Wright, L. D., Skeggs, H. R. \& Cresson, E. L. (1947). Proc. Soc. exp. Biol., N.Y., 64, 150.

Wright, L. D., Skeggs, H. R. \& Cresson, E. L. (1951). J. Amer. chem. Soc. 73, 4144.

Biochem. J. (1963) 89, 609

\title{
Avidin
}

\section{STABILITY AT EXTREMES OF pH AND DISSOCIATION INTO SUB-UNITS BY GUANIDINE HYDROCHLORIDE}

\author{
BY N. M. GREEN* \\ Department of Chemical Pathology, St Mary's Hospital Medical School, London, W. 2
}

(Received 19 March 1963)

The great stability of avidin over a wide $\mathrm{pH}$ range, particularly when combined with biotin, has frequently been noted and has been studied qualitatively by Fraenkel-Conrat, Snell \& Ducay $(1952 b)$. In the present paper a more detailed investigation was made by the technique of dif-

* Present address: Department of Chemistry, National Institute for Arthritis and Metabolic Diseases, Bethesda 14, Md., U.S.A. ference spectrophotometry. The behaviour of avidin in concentrated solutions of urea and guanidine was also examined since it appeared likely that the molecule was built up from sub-units (Melamed \& Green, 1963). The great stability, coupled with a molecular weight which was unchanged by $8 \mathrm{M}$ urea (Fraenkel-Conrat, Snell \& Ducay, 1952a), suggested that the sub-units might be linked covalently. Therefore attempts were made to separate

Bioch. 1963, 89 